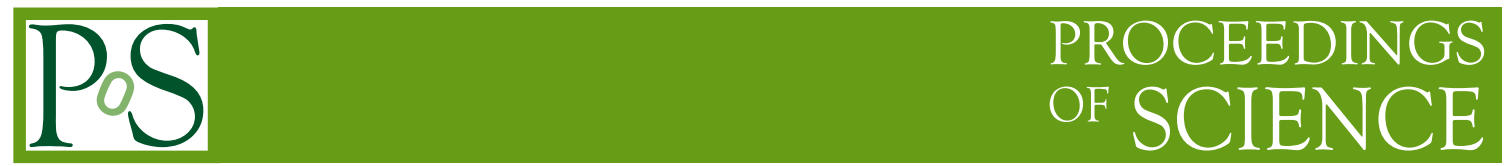

\title{
New approach to NN with perturbative pions
}

\section{Silas Beane*}

University of New Hampshire

E-mail: silas@physics.unh.edu

The current status of effective field theory (EFT) descriptions of nucleon-nucleon (NN) interactions is briefly reviewed, and a new formulation of EFT which treats pion interactions perturbatively is presented. This approach differs from the Kaplan-Savage-Wise (KSW) expansion in that the singular short distance part of the pion tensor interaction is summed to all orders.

6th International Workshop on Chiral Dynamics

July 6-10 2009

Bern, Switzerland

\footnotetext{
*Speaker.
} 


\section{Weinberg and KSW}

Weinberg was the first to describe an EFT for nuclear forces [1, 2, 3], and devised the prescription that one compute the nuclear potential in an EFT expansion, truncate at a given order, and then solve the Lippmann-Schwinger equation exactly with that potential. This program has since been pursued by a number of groups $[4,5,6,7,8,9,10,11,12,13,14,15,16,17,18,19,20]$, with very impressive fits to phase shift data at $N^{3} L O$. An advantage of this approach is that the long distance part of the interaction correctly incorporates chiral symmetry; furthermore, with Weinberg's power counting scheme for the EFT expansion, there is in principle a systematic improvement of the results with increasing order. A disadvantage of Weinberg's scheme is that in its naive implementation, there are divergences that cannot be absorbed by operators included at that order, arising from the singular nature of the EFT potential $[21,22,23]$. Thus results depend on a regulator scale $\Lambda$ which cannot be removed, implying that the treatment of short distance interactions is model-dependent. An analysis of high partial wave channels at NLO in the Weinberg EFT in ref. [23] demonstrated that the cutoff dependence was a feature of all channels subject to an attractive pion tensor force - despite the fact that there is no local operator to absorb this model dependence until order $(\ell+1)$ in the expansion for a channel with angular momentum $\ell$. Furthermore ref. [23] demonstrated that at this order, observables in channels with an attractive tensor interaction are particularly sensitive to the value of the cutoff even at energies as low as $T_{l a b}=50 \mathrm{MeV}$. It is argued that predictions at a given order only vary at the level of higher order corrections as the regulator is varied over some range, so that the model dependence does not interfere with the predictive power of the EFT. This hope is difficult to verify since the computations are all numerical, and the numerical evidence suggests that the acceptable range for $\Lambda$ is very narrow.

The alternative KSW theory entails an expansion of the NN scattering amplitude, instead of the nuclear potential, effected by computing a well-defined class of Feynman diagrams at each order in the expansion [21, 24, 25]. KSW power counting is not determined by how operators scale near the trivial IR fixed point of the nucleon contact interaction; instead it is determined by operator scaling about the nontrivial UV fixed point corresponding to infinite scattering length. At this fixed point nucleon operators for s-wave scattering develop large anomalous dimensions and are resummed nonperturbatively, a reasonable starting point given how much larger NN scattering lengths are than the range of their interaction.

The KSW scheme expands the NN scattering amplitude in powers of $Q$, where the nucleon momentum $p$, pion mass $m_{\pi}$ and the inverse scattering length $1 / a$ are all considered $O(Q)$, while other mass scales such as the nucleon mass $M$, the pion decay constant $f_{\pi}$ are taken to be $O(1)$. It was argued that convergence of the KSW expansion is governed by the scale $\Lambda_{N N}=16 \pi f_{\pi}^{2} /\left(g_{A}^{2} M\right)=$ $300 \mathrm{MeV}$. An advantage of this approach is that the scattering amplitudes can be computed analytically, and at each order the amplitude is renormalized and independent of the cutoff. NN phase shifts were computed to order NNLO in refs. [26, 27]; the result for the spin-singlet ${ }^{1} S_{0}$ phase shift is shown in Fig. 1, plotted versus the momentum $p$ of each nucleon in the center of mass frame.

Although successful in the spin-singlet channel, it was discovered in ref. [27] that the KSW expansion does not converge in the ${ }^{3} S_{1}$ channel, and the authors identified the singular tensor potential mediated by pions, scaling as $-1 / r^{3}$ for small $r$, to be the cause of the failure. Such a singular attractive interaction is incapable of supporting a ground state and no contact interaction 


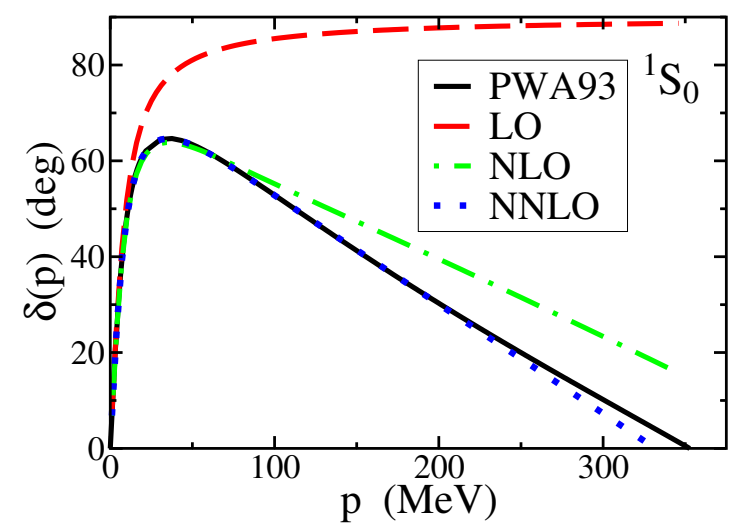

Figure 1: The ${ }^{1} S_{0} \mathrm{NN}$ phase shift in the KSW expansion, versus momentum in the center of mass frame to NNLO, compared with the Nijmegen PWA93 partial wave analysis [28].

can remedy this pathology. One possible solution suggested in [22] is to expand around the chiral limit, treating the infinite number of bound states in the pion potential as being short range and outside the realm of validity of the EFT.

\section{A New Expansion}

In ref. [29] we propose a different solution: we modify the pion propagator in a manner reminiscent of Pauli-Villars regulation characterized by a heavy mass scale $\lambda$. This modification removes the $1 / r^{3}$ singularity in pion exchange, effectively shifting that physics into the contact interactions and reordering the summation of strong short-distance effects. The advantages of the KSW expansion are retained: there is a well-defined power counting scheme that organizes the calculation, and results are analytic. Dependence on the scale $\lambda$ can therefore be studied analytically, and we find that all contributions that grow as powers of $\lambda$ are absorbed into counterterms. The limit $\lambda \rightarrow \infty$ is therefore smooth, and the KSW expansion is recovered in that limit. Here we present promising results for the low-lying spin triplet phase shifts to NNLO that indicate convergence of the expansion.

Our starting point is the assumption that the failure of the KSW expansion is due to the singular short distance pion tensor interaction, which can be eliminated by a shift in the contact interactions of the EFT. The underlying principles of EFT imply that we are free to distort the short range pion interactions however we please, as the counterterms serve to ensure the correct low energy effects of short distance physics. We therefore choose the modification in order to: (i) make it possible to analytically perform the diagrammatic expansion; (ii) leave unaltered the KSW expansion of the spin-singlet channel, since apparently no convergence problem is encountered there. These considerations lead us to replace the pion propagator $G_{\pi}\left(\mathbf{q}, m_{\pi}\right)$ by

$$
G_{\pi}\left(\mathbf{q}, m_{\pi}\right)+G_{(1,1)}(\mathbf{q}, \lambda)+G_{(1,0)}(\mathbf{q}, \lambda)
$$


where the subscript $(I, J)$ indicates the isospin and spin of a fictitious meson. Including couplings at the ends of the propagators, these expressions are given by

$$
\begin{aligned}
G_{\pi}\left(\mathbf{q}, m_{\pi}\right) & =i \frac{g_{A}^{2}}{4 f_{\pi}^{2}} \frac{\left(\mathbf{q} \cdot \boldsymbol{\sigma}_{1}\right)\left(\mathbf{q} \cdot \boldsymbol{\sigma}_{2}\right)\left(\boldsymbol{\tau}_{1} \cdot \boldsymbol{\tau}_{2}\right)}{\mathbf{q}^{2}+m_{\pi}^{2}} \\
G_{(1,0)}(\mathbf{q}, \lambda) & =i \frac{g_{A}^{2} \lambda^{2}}{4 f_{\pi}^{2}} \frac{\left(\boldsymbol{\tau}_{1} \cdot \boldsymbol{\tau}_{2}\right)}{\mathbf{q}^{2}+\lambda^{2}}
\end{aligned}
$$

and $G_{(1,1)}(\mathbf{q}, \lambda)=-G_{\pi}(\mathbf{q}, \lambda)$. The $G_{(1,1)}$ term looks like exchange of a pion with the wrong sign propagator and mass $\lambda$, canceling the short distance $1 / r^{3}$ part of the pion-induced tensor interaction for $r \lesssim 2 \pi / \lambda$. The $G_{(1,0)}$ term is included to exactly cancel $G_{(1,1)}$ (up to a contact interaction) in the spin-singlet channel; it resembles the exchange of an $I=1, J=0$ meson, also of mass $\lambda$. In the above expressions $g_{A} \simeq 1.25$ and $f_{\pi} \simeq 93 \mathrm{MeV} ; \boldsymbol{\sigma}$ and $\boldsymbol{\tau}$ are spin and isospin matrices respectively. Note that the only free parameter is the mass scale $\lambda$. We expect that for $\lambda \gtrsim 2 \Lambda_{N N}$ the derivative expansion is not adversely affected, and that the original KSW expansion is recovered in the $\lambda \rightarrow \infty$ limit.

We emphasize that we are not using $G_{(1,1)}$ and $G_{(1,0)}$ to model real meson exchange, but only as a device to eliminate the strong short distance behavior from the tensor pion exchange, putting all that physics in the contact interactions which are fit to data. Choosing the masses in $G_{(1,1)}$ and $G_{(1,0)}$ to both equal $\lambda$ greatly simplifies the analytic computations.

\section{NNLO calculation of spin-triplet amplitudes}

Making use of the modified pion propagator eq. (2.1) and classifying the mass scale $\lambda$ to also be $O(Q)$, we have computed all the Feynman diagrams in [26, 27] relevant for the ${ }^{3} S_{1},{ }^{3} D_{1}$ and $\varepsilon_{1}$ partial wave channels. These diagrams are evaluated using dimensional regularization and we choose the renormalization scale, $\mu=m_{\pi}$. The analytic formulas for our NNLO calculations will be given elsewhere; here we present the results graphically. In Fig. 2 we show our results with $\lambda=750 \mathrm{MeV}$ for the ${ }^{3} S_{1},{ }^{3} D_{1}$ and $\varepsilon_{1}$ phase shifts, compared with the Nijmegen partial wave analysis [28]. All three of our results are improvements over the NNLO KSW computation in [26, 27], and with the exception of $\varepsilon_{1}$, show signs of converging on the correct answer. The result for $\varepsilon_{1}$ is less convincing, but it should be noted that the anomalously small value for $\varepsilon_{1}$ in nature suggests that delicate cancellations are at play, and one would only expect an EFT prediction to start converging at high order in the expansion.

The dependence of our results on $\lambda$ is displayed in Fig. 3, where the bands indicate the changes in the phase shifts over the range $600 \mathrm{MeV} \leq \lambda \leq 1000 \mathrm{MeV}$. It is apparent from these figures that our results are not extremely sensitive at low $p$ to the value we take for $\lambda$.

The role of the scale $\lambda$ in these calculations can be easily addressed given the analytic form we have derived for the scattering amplitudes. It may seem strange that $\lambda-$ a regularization scale-is being treated as $O(Q)$ which is our low energy expansion scale. In particular, one might worry that scattering amplitudes have terms proportional to powers of $\lambda / \Lambda_{N N}$, which is formally $O(Q)$ but numerically $>1$. In fact though one can show analytically that at each order in the expansion, contributions to the amplitudes proportional to positive powers of $\lambda$ are all absorbed into the counterterms available at that order. Therefore the amplitudes only depend on inverse powers of 

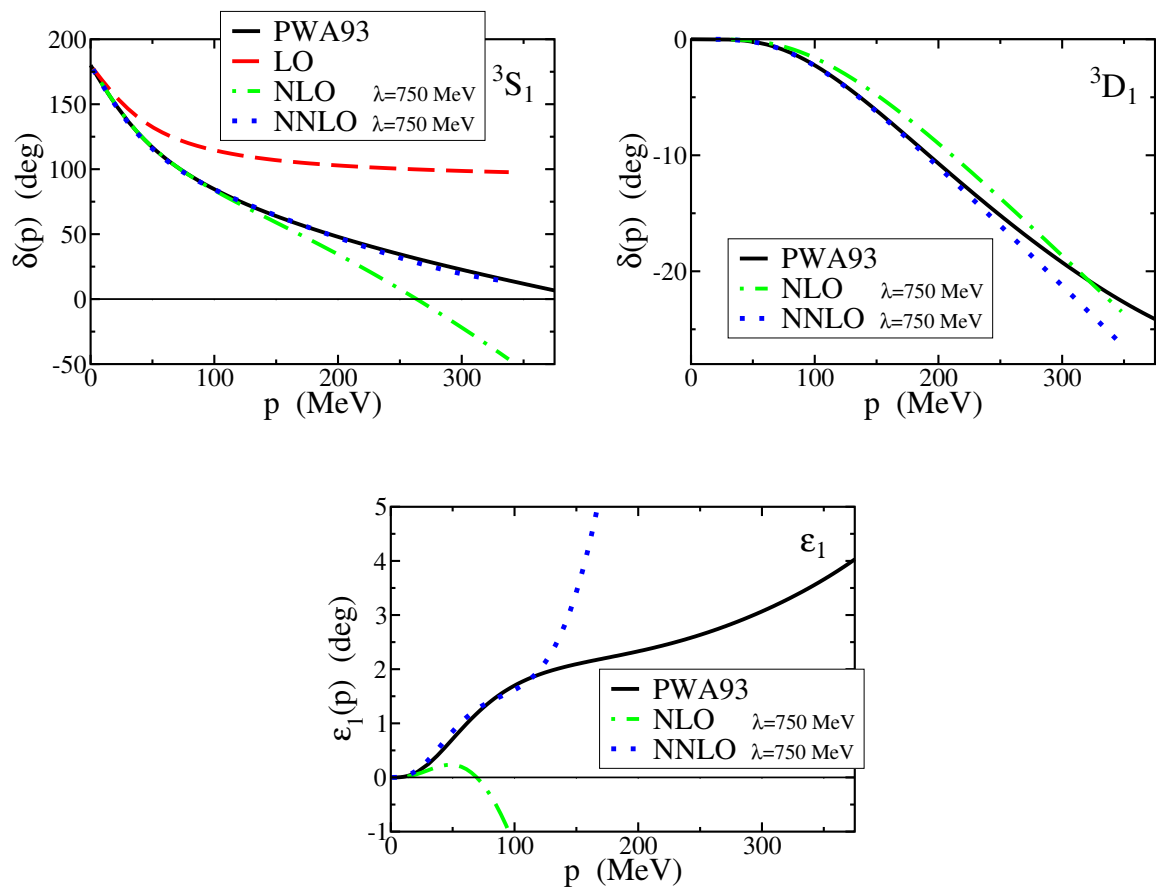

Figure 2: New results for the ${ }^{3} S_{1},{ }^{3} D_{1}$, and $\bar{\varepsilon}_{1}$ phase shifts plotted versus momentum in the center of mass frame to NNLO, compared with the Nijmegen PWA93 partial wave analysis.

$\lambda$, and in the $\lambda \rightarrow \infty$ limit the fictitious meson propagators in eq. (2.1) decouple and one smoothly recovers the results of $[26,27]$.

\section{Remaining issues}

The EFT scheme we have presented here for computing NN scattering in perturbation theory appears to converge well and preserve the desirable feature of the KSW scheme that at each order the amplitude can be computed as a well defined set of Feynman diagrams. Unlike the KSW scheme, there is now a new dimensionful parameter $\lambda$ which regulates the short distance tensor interaction. The manner with which we have performed this regulation is certainly not unique, and we have shown that our results are not particularly sensitive to the value of $\lambda$, and that over a wide range for $\lambda$ the variation of the phaseshifts are comparable to or smaller than higher order corrections in the EFT expansion.

On the other hand, we know that by taking $\lambda \rightarrow \infty$ we recover the KSW expansion, which fails to converge above $p \sim 100 \mathrm{MeV}$. The parameter $\lambda$ apparently plays a role analogous to the renormalization scale $\mu$ in perturbative QCD. The scale $\mu$ is unphysical, and a nonperturbative QCD calculation will not depend on it; however, at any finite order in perturbation theory, amplitudes do depend on $\mu$, and varying $\mu$ corresponds to reordering the perturbative expansion. Choosing $\mu$ appropriately (e.g., via the BLM scale-setting prescription [30]) can optimize the perturbative expansion, while non-optimal choices for $\mu$ lead to poor convergence. Similarly, $\lambda$ is an unphysical parameter, and varying $\lambda$ constitutes a reordering of the the EFT expansion, with smaller $\lambda$ 

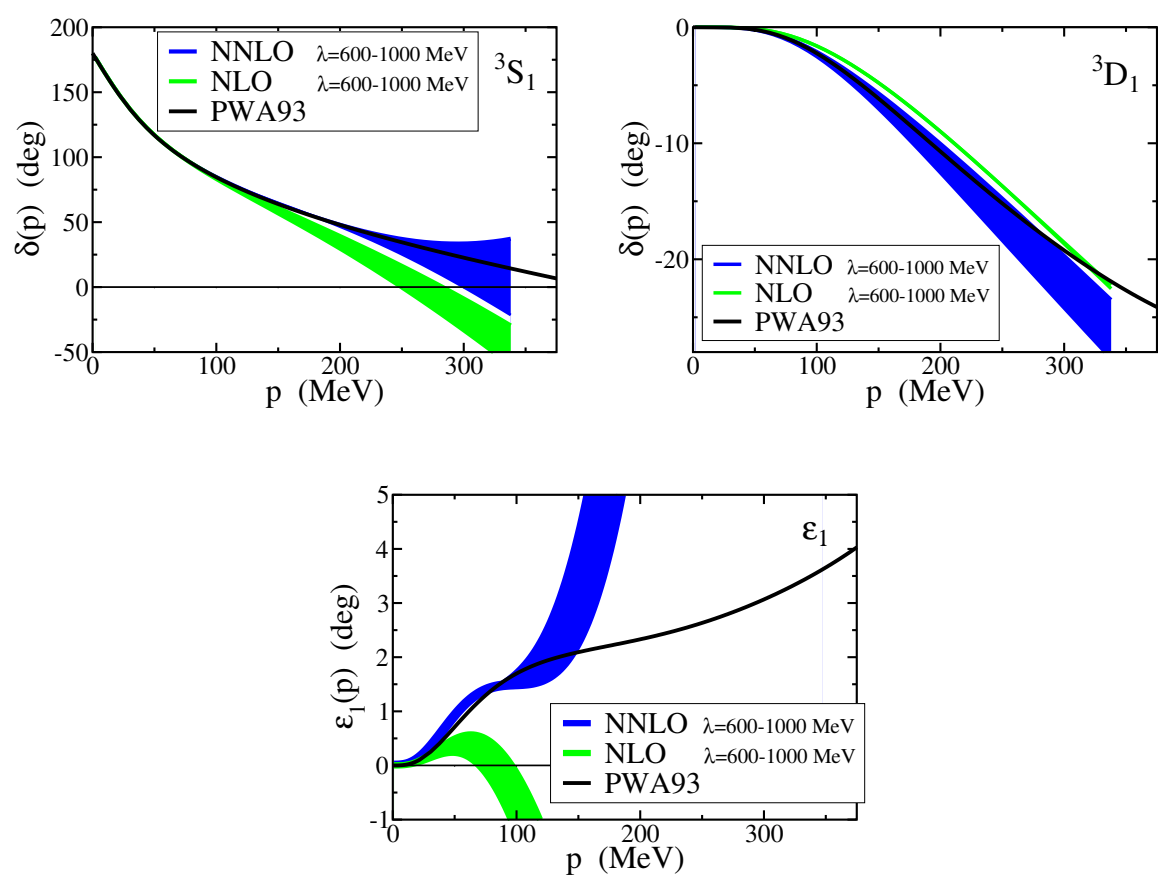

Figure 3: The NLO (green band) and NNLO (blue band) results for the ${ }^{3} S_{1},{ }^{3} D_{1}$, and $\bar{\varepsilon}_{1}$ phaseshifts showing their variation as $\lambda$ is varied in the range $600 \mathrm{MeV} \leq \lambda \leq 1000 \mathrm{MeV}$.

resulting in more of the pion interaction being accounted for in the resummed contact interactions. Taking $\lambda \simeq 750 \mathrm{MeV}$ appears to optimize the expansion, while choosing $\lambda=\infty$ yields the standard KSW expansion which fails to converge at relatively low momenta.

It is not possible to directly compare our expansion with the Weinberg expansion results at a given order, since the calculations are arranged differently. For example, one-pion and twopion exchange appear at $\mathrm{NLO}$ and $\mathrm{N}^{3} \mathrm{LO}$ in the KSW expansion respectively, while they appear at LO and NLO in the Weinberg expansion. Nevertheless, numerically our NNLO results compare favorably with the NLO Weinberg expansion results in [11], with the exception of $\varepsilon_{1}$ which is comparable to LO.

The perturbative EFT described here provides a well defined prescription for computing a number of additional processes to NNLO, such as electromagnetic effects, including form factors, Compton scattering, polarizabilities, and radiative capture, and it will be interesting to compare such results with experiment to thoroughly judge the efficacy of this method. An important outstanding issue is the generalization of the power counting to account for the higher partial waves. Evidently this poses a particularly difficult challenge to EFT descriptions of nuclear forces and the perturbative formulation described here is no exception. This issue will be addressed in future work.

\section{Acknowledgments}

I thank D.B. Kaplan and A. Vuorinen for a fruitful collaboration. This work was supported by NSF 
CAREER Grant No. PHY-0645570.

\section{References}

[1] S. Weinberg, Phys. Lett. B 251, 288 (1990).

[2] S. Weinberg, "Effective Chiral Lagrangians For Nucleon - Pion Interactions And Nuclear Nucl. Phys. B 363, 3 (1991).

[3] S. Weinberg, Phys. Lett. B 295, 114 (1992) [arXiv:hep-ph/9209257].

[4] C. Ordonez and U. van Kolck, Phys. Lett. B 291, 459 (1992).

[5] C. Ordonez, L. Ray and U. van Kolck, Phys. Rev. Lett. 72, 1982 (1994).

[6] U. van Kolck, Phys. Rev. C 49, 2932 (1994).

[7] C. Ordonez, L. Ray and U. van Kolck, Phys. Rev. C 53, 2086 (1996) [arXiv:hep-ph/9511380].

[8] J. L. Friar, D. Huber and U. van Kolck, Phys. Rev. C 59, 53 (1999) [arXiv:nucl-th/9809065].

[9] M. C. M. Rentmeester, R. G. E. Timmermans, J. L. Friar and J. J. de Swart, Phys. Rev. Lett. 82, 4992 (1999) [arXiv:nucl-th/9901054].

[10] V. Bernard, N. Kaiser and U. G. Meissner, "Determination of the low-energy constants of the next-to-leading order Nucl. Phys. A 615, 483 (1997) [arXiv:hep-ph/9611253].

[11] E. Epelbaum, W. Gloeckle and U. G. Meissner, "Nuclear forces from chiral Lagrangians using the method of unitary Nucl. Phys. A 671, 295 (2000) [arXiv:nucl-th/9910064].

[12] E. Epelbaum, H. Kamada, A. Nogga, H. Witala, W. Gloeckle and U. G. Meissner, Phys. Rev. Lett. 86, 4787 (2001) [arXiv:nucl-th/0007057].

[13] E. Epelbaum, A. Nogga, W. Gloeckle, H. Kamada, U. G. Meissner and H. Witala, "Few nucleon systems with two-nucleon forces from chiral effective field Eur. Phys. J. A 15, 543 (2002) [arXiv:nucl-th/0201064].

[14] E. Epelbaum, W. Gloeckle and U. G. Meissner, "Improving the convergence of the chiral expansion for nuclear forces. II: Eur. Phys. J. A 19, 401 (2004) [arXiv:nucl-th/0308010].

[15] E. Epelbaum, W. Glockle and U. G. Meissner, Nucl. Phys. A 747, 362 (2005) [arXiv:nucl-th/0405048].

[16] D. R. Entem and R. Machleidt, "Accurate nucleon nucleon potential based upon chiral perturbation Phys. Lett. B 524, 93 (2002) [arXiv:nucl-th/0108057].

[17] D. R. Entem and R. Machleidt, Phys. Rev. C 66, 014002 (2002) [arXiv:nucl-th/0202039].

[18] D. R. Entem and R. Machleidt, "Accurate Charge-Dependent Nucleon-Nucleon Potential at Fourth Order of Phys. Rev. C 68, 041001 (2003) [arXiv:nucl-th/0304018].

[19] M. Pavon Valderrama and E. R. Arriola, "Renormalization of NN Interaction with Chiral Two Pion Exchange Potential. Phys. Rev. C 74, 054001 (2006) [arXiv:nucl-th/0506047].

[20] M. Pavon Valderrama and E. Ruiz Arriola, "Renormalization of NN Interaction with Chiral Two Pion Exchange Potential. Phys. Rev. C 74, 064004 (2006) [Erratum-ibid. C 75, 059905 (2007)] [arXiv:nucl-th/0507075].

[21] D. B. Kaplan, M. J. Savage and M. B. Wise, Nucl. Phys. B 478, 629 (1996) [arXiv:nucl-th/9605002]. 
[22] S. R. Beane, P. F. Bedaque, M. J. Savage and U. van Kolck, Nucl. Phys. A 700, 377 (2002) [arXiv:nucl-th/0104030].

[23] A. Nogga, R. G. E. Timmermans and U. van Kolck, Phys. Rev. C 72, 054006 (2005) [arXiv:nucl-th/0506005].

[24] D. B. Kaplan, M. J. Savage and M. B. Wise, Nucl. Phys. B 534, 329 (1998) [arXiv:nucl-th/9802075].

[25] D. B. Kaplan, M. J. Savage and M. B. Wise, Phys. Lett. B 424, 390 (1998) [arXiv:nucl-th/9801034].

[26] S. Fleming, T. Mehen and I. W. Stewart, Phys. Rev. C 61, 044005 (2000) [arXiv:nucl-th/9906056].

[27] S. Fleming, T. Mehen and I. W. Stewart, Nucl. Phys. A 677, 313 (2000) [arXiv:nucl-th/9911001].

[28] V. G. J. Stoks, R. A. M. Kompl, M. C. M. Rentmeester and J. J. de Swart, "Partial wave analaysis of all nucleon-nucleon scattering data below Phys. Rev. C 48, 792 (1993).

[29] S. R. Beane, D. B. Kaplan and A. Vuorinen, Phys. Rev. C 80, 011001 (2009) [arXiv:0812.3938 [nucl-th]].

[30] S. J. Brodsky, G. P. Lepage and P. B. Mackenzie, "On The Elimination Of Scale Ambiguities In Perturbative Quantum Phys. Rev. D 28, 228 (1983). 